Editorial

\title{
Advanced Intelligent Technologies in Energy Forecasting and Economical Applications
}

\author{
Wei-Chiang Hong $\mathbb{D}^{1},{ }^{1}$ Dongxiao Niu, ${ }^{2}$ Yinfeng $\mathrm{Xu},{ }^{3}$ Mengjie Zhang, \\ and Pradeep Kumar Singh \\ ${ }^{1}$ Asia Eastern University of Science and Technology, New Taipei City, Taiwan \\ ${ }^{2}$ North China Electric Power University, Beijing, China \\ ${ }^{3}$ Donghua University, Shanghai, China \\ ${ }^{4}$ Victoria University of Wellington, Wellington, New Zealand \\ ${ }^{5}$ KIET Group of Institutions, Delhi-NCR, Ghaziabad, U.P., India \\ Correspondence should be addressed to Wei-Chiang Hong; samuelsonhong@gmail.com
}

Received 27 November 2021; Accepted 27 November 2021; Published 9 December 2021

Copyright (c) 2021 Wei-Chiang Hong et al. This is an open access article distributed under the Creative Commons Attribution License, which permits unrestricted use, distribution, and reproduction in any medium, provided the original work is properly cited.

Accurate energy forecasting is essential to achieve greater efficiency and reliability in power system operation and security, energy pricing problems, and scheduling and planning of energy supply systems, among other areas. Particularly, in the Big Data era, forecasting models are always based on a complex function combination, and energy data are always complicated; examples include seasonality, cyclicity, fluctuation, and dynamic nonlinearity. During the past few decades, many energy forecasting models have been proposed, including traditional statistical models and artificial intelligent models. However, most of these models often possess theoretical drawbacks which limit their forecasting performance. These forecasting models have resulted in an over-reliance on the use of informal judgments and higher expenses when lacking the ability to determine data characteristics and patterns.

Recently, due to the development of advanced intelligent computing technologies, many novel technologies hybridised or combined with the energy forecasting and economic planning models mentioned previously have received much attention. The hybridization of optimization methods and superior evolutionary algorithms can provide important improvements via well parameter determinations in the optimization process, which is of great assistance to actions taken by energy decision-makers. It is important to explore the development of the modeling methodology by applying these advanced intelligent technologies.

The aim of this special issue is to collate original research articles with a focus on the applications of advanced intelligent technologies in economical modeling and energy forecasting. After a rigorous round of doubleblind peer review process, finally 10 papers are accepted for publication in this special issue, which all display a broad range of cutting edge topics in the advanced intelligent technologies. The editors of this special issue believe that the advanced intelligent technologies and computation techniques will play more important role in energy forecasting accuracy improvements to overcome some critical shortcomings of a single model or directly improve the shortcoming by theoretical innovative arrangements.

The first paper is "Time Series Prediction of Electricity Demand Using Adaptive Neuro-Fuzzy Inference Systems" by Acakpovi et al.; they concerned with the reliable prediction of electricity demands by using the adaptive neurofuzzy inference system (ANFIS). Based on the experimental analysis and the comparison results, the performance of the avalanche photodiode is around $11 \%$ better than the pin diode.

The second paper is "A Comparison of Hour-Ahead Solar Irradiance Forecasting Models Based on LSTM 
Network" by Huang et al.; they investigated the Model I-A and Model II-B based on traditional long short-term memory (LSTM) and the effects of different parameters. With real data over 5 years, the experimental results demonstrate that Model II-BD shows the best performance because it considers the weather information of the next moment, the root mean square error (RMSE) is $62.1618 \mathrm{~W} /$ $\mathrm{m}^{2}$, the normalized root mean square error (nRMSE) is $32.2702 \%$, and the forecast skill (FS) is 0.4477 , which is $19.19 \%$ more accurate than the backpropagation neural network (BPNN) in terms of RMSE.

The third paper is "Optimal Control and Simulation for Enterprise Financial Risk in Industry Environment" by Liang et al.; they developed a technique for enterprise financial risk optimal control with exponential decay rate and simulation in industry environment. The factors of industry environment risks to enterprise financial activities are considered; seven kinds of industry environment risks influencing enterprise financial activities are chosen as state variables. Numerical simulation results illustrate the effectiveness of the proposed technique.

The fourth paper is "Optimizing the Procurement of IaaS Reservation Contracts via Workload Predicting and Integer Programming" by Zhu et al.; they investigated the problem of how to minimize IaaS rental cost associated with hosting web applications, while meeting the demand in the future business cycle, by using integer liner program model and a long short-term memory (LSTM). The experimental prediction results show the LSTM-based algorithm gains an advantage over several popular models, such as the Holter-Winters, the seasonal autoregressive integrated moving average (SARIMA), and the support vector regression (SVR).

The fifth paper is " $\mathrm{CO}_{2}$ Emissions, Energy Consumption, and Economic Growth Nexus: Evidence from 30 Provinces in China" by Zou and Zhang; they established a spatial Durbin model including economic growth, energy consumption equation, and $\mathrm{CO}_{2}$ emissions and studied the dynamic relationship and spatial spillover among economic growth, energy consumption, and $\mathrm{CO}_{2}$ emissions effects. The results show that the economic growth can significantly improve carbon dioxide emissions, and China's economic growth level has become a positive driving force for carbon dioxide emissions. However, economic growth will not be significantly affected by the reduction of carbon dioxide emissions, and energy consumption and carbon emissions are interrelated, which has a negative spatial spillover effect on the carbon dioxide emissions of the surrounding provinces and cities.

The sixth paper is "The Improved Least Square Support Vector Machine Based on Wolf Pack Algorithm and Data Inconsistency Rate for Cost Prediction of Substation Projects" by Wang et al.; they proposed a forecasting model based on the improved least squares support vector machine (ILSSVM) optimized by wolf pack algorithm (WPA) to improve the accuracy and stability of the cost forecasting of substation projects. They selected 88 substation projects in different regions from 2015 to 2017 to conduct the training tests to verify the validity of the model. The results indicate that the new hybrid WPA-DIR-ILSSVM model presents better accuracy, robustness, and generality in cost forecasting of substation projects.

The seventh paper is "A Novel Hybrid Approach Based on BAT Algorithm with Artificial Neural Network to Forecast Iran's Oil Consumption" by Bahmani et al.; they developed a function of population, GDP, import, and export by applying a hybrid bat algorithm (BAT) and artificial neural network (ANN) to forecast oil consumption in Iran. The result of the model shows that the findings are in close agreement with the observed data, and the performance of the method was excellent. Authors demonstrate that its efficiency could be a helpful and reliable tool for monitoring oil consumption.

The eighth paper is "Nonlinear Effect Analysis of Electricity Price on Household Electricity Consumption" by Zhang and Wen; they investigated the scenario effect of per capita income and regional differences in urbanization development on the relationship between electricity sales price and urban household electricity consumption. They discussed the effect of electricity sales price on urban household electricity consumption from the perspective of regional difference in income and urbanization, which provides the decision-making basis and empirical support for developing regional electricity price policy and household energy consumption policy.

The ninth paper is "The Impact and Stability Analysis of Commercial Banks' Risk Preference on SMEs' Credit Financing Based on DSGE Model" by Gao et al.; they analyzed the impact of financial intermediary departments' risk preference on corporate finance. Under the revised DSGE framework, they discussed the impact and stability analysis of commercial banks' risk preferences on SMEs' financing. The results showed that positive interest rate shocks inhibit commercial banks' credit to SMEs, and with the increasing weight of commercial banks' risk preference for default rate, the trend of credit repression will be intensified.

The tenth paper is "Evolutionary Framework with Bidirectional Long Short-Term Memory Network for Stock Price Prediction" by Zheng et al.; they proposed a novel bidirectional long short-term memory network (BiLSTM) framework called evolutionary BiLSTM (EBiLSTM) for the prediction of stock price. Experiments on several stock market indexes demonstrate that EBiLSTM can achieve better prediction performance than others without the evolutionary operator.

\section{Conflicts of Interest}

The editors declare that they have no conflicts of interest regarding the publication of this special issue.

\author{
Wei-Chiang Hong \\ Dongxiao Niu \\ Yinfeng $X u$ \\ Mengjie Zhang \\ Pradeep Kumar Singh
}

